\title{
Multiwavelength Properties of the X-ray Sources in the Groth-Westphal Strip Field ${ }^{1}$
}

\author{
Takamitsu Miyaji ${ }^{1}$, Vicki Sarajedini² ${ }^{2}$ Richard E. Griffiths ${ }^{1}$, Toru Yamada ${ }^{3}$, Matthew \\ Schurch $^{1,4}$, David Cristóbal-Hornillos ${ }^{5}$, Kentaro Motohara ${ }^{6}$
}

\begin{abstract}
We summarize the multiwavelength properties of X-ray sources detected in the $80 \mathrm{ks}$ XMMNewton observation of the Groth-Westphal Strip, a contiguous strip of 28 HST Wide-Field Planetary Camera 2 (WFPC2) images. Among the $\approx 150$ X-ray sources detected in the XMM-Newton field of view, 23 are within the WFPC2 fields. Ten spectroscopic redshifts are available from the Deep Extragalactic Evolutionary Probe (DEEP) and Canada-France Redshift Survey (CFRS) projects. Four of these show broad Mg II emission and can be classified as type 1 AGNs. Two of those without any broad lines, nevertheless, have $[\mathrm{NeV}]$ emission which is an unambiguous signature of AGN activity. One is a narrow-line Seyfert 1 and the other a type 2 AGN.

As a followup, we have made near-infrared (NIR) spectroscopic observations using the OHS/CISCO spectrometer for five of the X-ray sources for which we found no indication of an AGN activity in the optical spectrum. We have detected $\mathrm{H} \alpha+[\mathrm{NII}]$ emission in four of them. A broad $\mathrm{H} \alpha$ component and/or a large [NII]/ $\mathrm{H} \alpha$ ratio is seen, suggestive of AGN activity. Nineteen sources have been detected in the $K_{\mathrm{s}}$ band and four of these are extremely red objects (EROs; $I_{814}-K_{\mathrm{s}}>4$ ).

The optical counterparts for the majority of the X-ray sources are bulge-dominated. The $I_{814}-K_{\mathrm{s}}$ color of these bulge-dominated hosts are indeed consistent with evolving elliptical galaxies, while contaminations from star formation/AGN seems to be present in their $V_{606}-I_{814}$ color. Assuming that the known local relations among the bulge luminosity, central velocity dispersion, and the mass of the central blackhole still hold at $z \sim 1$, we compare the AGN luminosity with the Eddington luminosity of the central blackhole mass. The AGN bolometric luminosity to Eddington luminosity ratio ranges from 0.3 to $10 \%$.
\end{abstract}

Subject headings: galaxies: bulges - (galaxies:) quasars: general - galaxies: Seyfert - X-rays: galaxies $\mathrm{X}$-rays: general

\footnotetext{
${ }^{1}$ Department of Physics, Carnegie Mellon University, Pittsburgh, PA 15213 (miyaji@cmu.edu griffith@seren.phys.cmu.edu)

${ }^{2}$ Department of Astronomy, University of Florida, P.O. Box 112055, Gainesville, FL 32611 (vicki@astro.ufl.edu)

${ }^{3}$ National Astronomical Observatory of Japan, Mitaka 181-8588, Japan; yamada@optik.mtk.nao.ac.jp

${ }^{4}$ Department of Physics, U. Bristol, Tyndall Avenue, Bristol BS8 1TL, UK

${ }^{5}$ Instituto de Astrofísica de Canarias, 38200 La Laguna, Tenerife, Spain

${ }^{6}$ Institute of Astronomy, University of Tokyo, Mitaka, Tokyo 181-0015, Japan

${ }^{1}$ Based on observations from the XMM-Newton, an ESA
}

\section{Introduction}

Deep X-ray images of a patch of the sky show numerous X-ray sources, which mainly consist of a mixture of absorbed and unabsorbed active galactic nuclei (AGNs). It is now recognized that these AGNs make up the bulk of what has been called the "X-ray Background". While normal galaxies, whose X-ray emission is probably dom-

science mission with instruments and contributions directly funded by ESA member states and the USA (NASA). Also based on data collected at Subaru Telescope, which is operated by the National Astronomical Observatory of Japan. 
inated by the integration of X-ray binaries, start to emerge (Miyaji \& Griffiths 2002; Hornschemeier et al. 2003) at the faintest fluxes, the dominant Xray source population in the deep Chandra and XMM-Newton Surveys comes from AGN activities. Thus multiwavelength studies of these $\mathrm{X}$ ray sources are key to understanding the detailed history and physical conditions of the formation and the growth of the supermassive blackholes (SMBHs), which are now known to reside in the centers of almost all galaxies with a bulge (Magorrian et al. 1998; Merritt \& Ferrarese 2001).

The region known as the "Groth-Westphal Strip" (GWS), consists of Hubble Space Telescope (HST) Wide-Field Planetary Camera 2 (WFPC2) medium-deep images of a strip of 28 contiguous fields(Groth et al. 1994). It is a particularly useful field for extensive multiwavelength studies. $\mathrm{Nu}$ merous on-going and future followup projects have been/ are being conducted on and around this field. Existing morphological information from the original WFPC2 observations, combined with the Deep Extragalactic Evolutionary Probe (DEEP) ${ }^{2}$, Canada-France Redshift Survey (CFRS) ${ }^{3}$ and the ongoing DEEP $2{ }^{4}$ redshift surveys, is providing us with the first clues to the nature of the X-ray source counterparts.

Because of the contamination from starlight in the host galaxy, optical searches for faint AGNs in deep survey fields such as GWS need elaborate efforts. Such attempts have been made by searching for an unresolved component at the centers of galaxies, searching for variable nuclei, and by selecting those with ultraviolet-excess cores (Sarajedini, Green, Griffiths, \& Ratnatunga 1999; Sarajedini, Gilliland, \& Kasm 2003; Beck-Winchatz \& Anderson 1999). These surveys reveal up to $10 \%$ of galaxies as AGN candidates with nuclei extending as faint as $\mathrm{M}_{B} \simeq-15$. Optical searches, however, are less sensitive to AGNs obscured by dust around the active nucleus. On the other hand, X-ray surveys for AGNs are not hindered by the luminosity of the underlying host galaxy. In particular, X-ray surveys with hard band $(E>2$ $\mathrm{keV}$ ) sensitivity are also sensitive to the obscured AGNs.

\footnotetext{
${ }^{2}$ http://deep.ucolick.org/

${ }^{3}$ http://www.astro.utoronto.ca/ lilly/CFRS/

${ }^{4}$ http://deep.berkeley.edu/
}

In view of this, we have obtained an $80 \mathrm{ks}$ exposure the northeast part of the GWS with XMMNewton. This field also has the advantage of a low column density of neutral gas in our galaxy, corresponding to $N_{\mathrm{H}}=1.310^{20} \mathrm{~cm}^{-2}$ (Dickey \& Lockman 1990). A quick look view and the preliminary Log N-Log S relations from these data have been presented in Miyaji \& Griffiths (2001) and Miyaji et al. (2003). In this paper, we present the nature of the $23 \mathrm{X}$-ray sources in the GWS, where morphological properties from the WFPC2 images and some redshifts are available from the DEEP/CFRS redshift surveys. We further make supplemental near infrared spectroscopic observation for some of these X-ray sources for which we did not observe AGN signatures in the optical spectra. $K_{\mathrm{s}}$ band photometry of the X-ray sources are also presented.

The scope of this paper is as follows. In Section 2, we describe the X-ray data and analysis, including source detection and spectral analysis. In Section 3, we explain the source of the optical and near infrared (NIR) data. The optical and NIR nature of the XMM-Newton sources and related statistics are presented. In 4 , we discuss the overall results on these X-ray sources and black hole mass and the relationship with the bulge luminosity. We also comment on selected individual sources. A summary is given in 5. Throughout this paper, we use $H_{0}=70 \mathrm{~km} \mathrm{~s}^{-1} \mathrm{Mpc}^{-1}$, $\Omega_{\mathrm{m}}=0.3$, and $\Omega_{\Lambda}=0.7$. Unless otherwise noted, $L_{\mathrm{x}}$ is the $2-10 \mathrm{keV}$ rest-frame luminosity in units of $\mathrm{erg} \mathrm{s}^{-1}$ calculated using the cosmological parameters shown above.

\section{X-ray Data and Analysis}

The field has been observed with XMM-Newton as part of a Guaranteed Time program (PI: Griffiths). After cleaning for background flares, we have obtained a total of $69 \mathrm{ks}$ and $81 \mathrm{ks}$ of exposure for EPIC PN and MOS respectively. The log of observation is shown in Table 1.

Table 1: Log of XMM-Newton Observations

\begin{tabular}{cll}
\hline \hline Rev/OBSID & cleaned exp. & Obs. Date \\
\hline $0113 / 0127921001$ & $51 .(\mathrm{PN}) / 56 .(\mathrm{MOS})$ & 21-Jul.-2000 \\
& & \\
$\begin{array}{c}0114 / 0127921101 \\
\text { " } / 0127921201\end{array}$ & 18.(PN)/25.(MOS) & 23-Jul.-2000 \\
\hline
\end{tabular}


Firstly we have generated an X-ray image for each of the PN, MOS1, and MOS2 data from XMM-Newton revolutions 0113 and 0114 . We have determined the positions of the counterpart X-ray sources by Gaussian fits for two broad line AGNs detected in the Canada-France Redshift Survey (Lilly, Hammer, Le Fevre, \& Crampton 1995) and a few objects in the Medium Deep Survey (MDS) database (Ratnatunga, Griffiths, \& Ostrander 1999) with trivial X-ray counterparts. Based on the positions of the optical counterparts, we have determined the linear transformation coefficients of the X-ray source positions. During this process, we did not use X-ray sources which fell in the gaps of the CCDs. Using the coefficients, we then aligned each cleaned EPIC event file.

Using the aligned events, we made summed images in three energy bands, corresponding to photon energies of 0.5-2, 2-4.5 and 4.5-10 keV. We also made a $2-10 \mathrm{keV}$ image. In making the images, we have used PATTERN $\leq 4$ (12) for the PN (MOS) data and applied the standard screening criteria. In order to avoid the instrumental line emission feature which dominates the background in 7.33$9.05 \mathrm{keV}$, the channel energy range corresponding to this energy range has been excluded from the PN data. Also exposure maps have been created for each energy band using the task "eexpmap". The MOS exposure maps in each band have been multiplied by the ratio of the MOS and PN efficiencies in the given band, so that the source counts from the summed ( $\mathrm{PN}+2 \mathrm{MOSs})$ image divided by the summed exposure map gives count rates which can be converted to fluxes using the $\mathrm{PN}$ response. The slight shift due to the alignment process (at most $2^{\prime \prime}$ ) is negligible in the usage of the exposure map.

The final source detection has been made to the sum of all PN and MOS data for the three bands. These three images were simultaneously fed into the SAS software 'eboxdetect' in the "local" mode. For each band, we have created the "background map" by cubic spline fitting the source excluded image (divided by the exposure map) using the sources detected in the first step. The next step is to use these "background" maps to re-run the 'eboxdetect' in the "map" mode, i.e., search for the excess over the background map. The final step is to make a multi-source maximum-likelihood fit over the background map using the positions of the sources in the previous step as a starting model. This has been made using the SAS task "emldetect". The images in the soft $(0.5-2 \mathrm{keV})$, medium (2-4.5 keV), and ultra-hard (4.5-10 keV) bands have been simultaneously fit. The procedure also calculates the source detection likelihood for each band as well as for the sum of these. We accepted sources with a likelihood parameter of

$$
M L=-\ln (1-P)>14
$$

where $P$ is the probability that the source exists. We also made a separate source detection to the $2-10 \mathrm{keV}$ image to obtain fluxes of the sources in a more traditional energy band.

One of the caveats is that cubic spline fits generated using the SAS procedure "esplinemap" sometimes generate spurious wavy structures depending on the choice of the number of spline nodes (the esplinemap parameter nsplinenodes). This is probably because some structures in the input image are not well represented by the spline function for the given number of nodes. In order to obtain the best background map, we have manually varied the number of spline nodes until a satisfactory background map is obtained. While this strategy involves a certain subjectiveness, we have checked the goodness of this strategy with extensive simulations. The basic results of this analysis are presented in Miyaji et al. (2003). While the source detection should be improved for further quantitative analysis (Baldi et al. 2002; Valtchanov, Pierre, \& Gastaud 2001), our current source detection scheme provides a sufficiently complete list of Xray sources for the purpose of this paper which is focused on the multiwavelength properties of the X-ray sources within a limited area. These have been verified by visual comparisons of the images and the detected source lists. The detected source counts have been converted into "effective" count rates by dividing by the value of the exposure map smoothed with a $\sigma=12^{\prime \prime}$ gaussian.

In this paper, we concentrate on the sources which fall within the HST WFPC2 fields of view. Sixteen of the original 28 GWS fields overlap with the XMM field of view. Additionally, there is another WFPC2 field in this general field (PI Lilly). We excluded from this paper a few X-ray sources located close to the edge of the WFPC2 field if their optical counterparts were not included in the MDS database. Table 2 lists the X-ray sources 
within the WFPC2 FOVs detected using the above procedure. In this table, the fluxes have been obtained from the count rate assuming a power-law or an absorbed power-law spectrum, depending on the hardness ratios from detected count rates in the $0.5-2$ and $2-10 \mathrm{keV}$ bands. If the hardness ratio corresponds to an effective photon index of $\Gamma>1.8$, a power-law spectrum is used. For a harder spectrum, an absorbed spectrum with fixed $\Gamma=1.8$ and varying intrinsic absorption column density $\left(N_{\mathrm{H}}\right)$ placed at $z=1$ (a typical redshift of the sources) is used. As long as the value of $N_{\mathrm{H}}$ is matched to the observed hardness ratio, the count rate to flux conversion factor is insensitive to the assumed redshift.

The columns of Table 2 are explained below:

X-No.: The internal XMM source ID (hereafter referred to as the $\mathrm{X}$-number), which is used to identify the XMM-Newton sources throughout this paper (with or without the preceding " $\mathrm{x}$ ").

Name: The source name according to the IAU registry in the format XMMGWS JHHMMSS.s+DDMMSS. This also gives the detected XMM source position. Note that the last digits are truncated. ${ }^{5}$.

Pos. err: Positional error (90\% encircled) in arcseconds. See below.

XMM counts: The detected counts in the total (0.5-10 keV) band.

$\mathbf{S}_{\mathrm{x} 14}(\mathbf{0 . 5}-\mathbf{2})$ : The soft $(0.5-2 \mathrm{keV})$ band flux, in units of $10^{-14} \mathrm{erg} \mathrm{s}^{-1} \mathrm{~cm}^{-2}$.

$\mathbf{S}_{\mathrm{x} 14}(\mathbf{2}-\mathbf{1 0})$ : The hard $(2-10 \mathrm{keV})$ band flux, in the same units.

$\mathbf{M L}_{\text {tot }}$ : The source existence likelihood (see above) in the total band.

$\mathbf{M L}_{\mathrm{sft}}$ : The source existence likelihood in the soft band.

$\mathbf{M L}_{\mathrm{hrd}}$ : The source existence likelihood in the hard band.

\footnotetext{
${ }^{5}$ See http://cdsweb.u-strasbg.fr/iau-spec.html)
}

While we treated sources with $M L>14$ for the total band as detected and included them in Table 2 , in each of the soft and hard bands, the $M L$ values are smaller. In the soft and hard bands, we list a nominal flux and $1 \sigma$ error if $M L \geq 10$ and a $3 \sigma$ upper limit for a lower significance.

Fig. 1 shows the summed full-band (0.5-10 $\mathrm{keV)}$ XMM-Newton image smoothed with a $\sigma=$ $2^{\prime \prime}$ Gaussian for display. The HST WFPC2 FOVs are overlaid and the positions of the sources in Table 2 are marked with crosses with the $\mathrm{X}$-number designations.

There is a $200 \mathrm{ks}$ Chandra ACIS-I observation (PI Nandra) covering the northeastern part of the field and the data came to public archive immediately after the observation. We have also generated a source list from the Chandra data. We have aligned the Chandra events in the same way as the XMM-Newton data. Within 7.4 arcminutes of the Chandra pointing center, the Chandra data provide much more accurate $\mathrm{X}$-ray source positions and we used them for unambiguous identification of the X-ray source counterparts as shown below. In this paper, we choose not to discuss Xray sources detected in the Chandra data but not detected in the XMM-Newton data, because they are fainter than those in this paper and the current optical data and redshift information are very limited.

The uncertainties of the source positions have been determined by the combination of the empirically determined systematic errors and the statistical errors. The former has been determined by the rms residuals of the alignment procedure described above. These are $\sigma_{\text {sys }}=1 . " 5$ for the XMM data and 0 !'7 for the Chandra data along each of the $\mathrm{x}$ and $\mathrm{y}$ axes. For fainter sources and/or sources with large off-axis angles, the statistical error of the source position $\left(\sigma_{\text {stat }}\right)$ becomes comparable to $\sigma_{\text {sys }}$. Since the $\sigma_{\text {stat }}$ is much smaller than $\sigma_{\text {sys }}$ for brighter sources, including those used for the alignment process, $\sigma_{\text {stat }}$ and $\sigma_{\text {sys }}$ are independent of each other. Thus we can estimate the $90 \%$ encircle radius $\left(r_{0.9}\right)$ by

$$
r_{0.9}=2.15\left[\sigma_{\text {sys }}^{2}+\sigma_{\text {stat }}^{2}\right]
$$

where 2.15 is the conversion factor between $1 \sigma$ and $90 \%$ encircle radius assuming a two-dimensional Gaussian probability distribution.

The XMM X-ray sources with improved posi- 


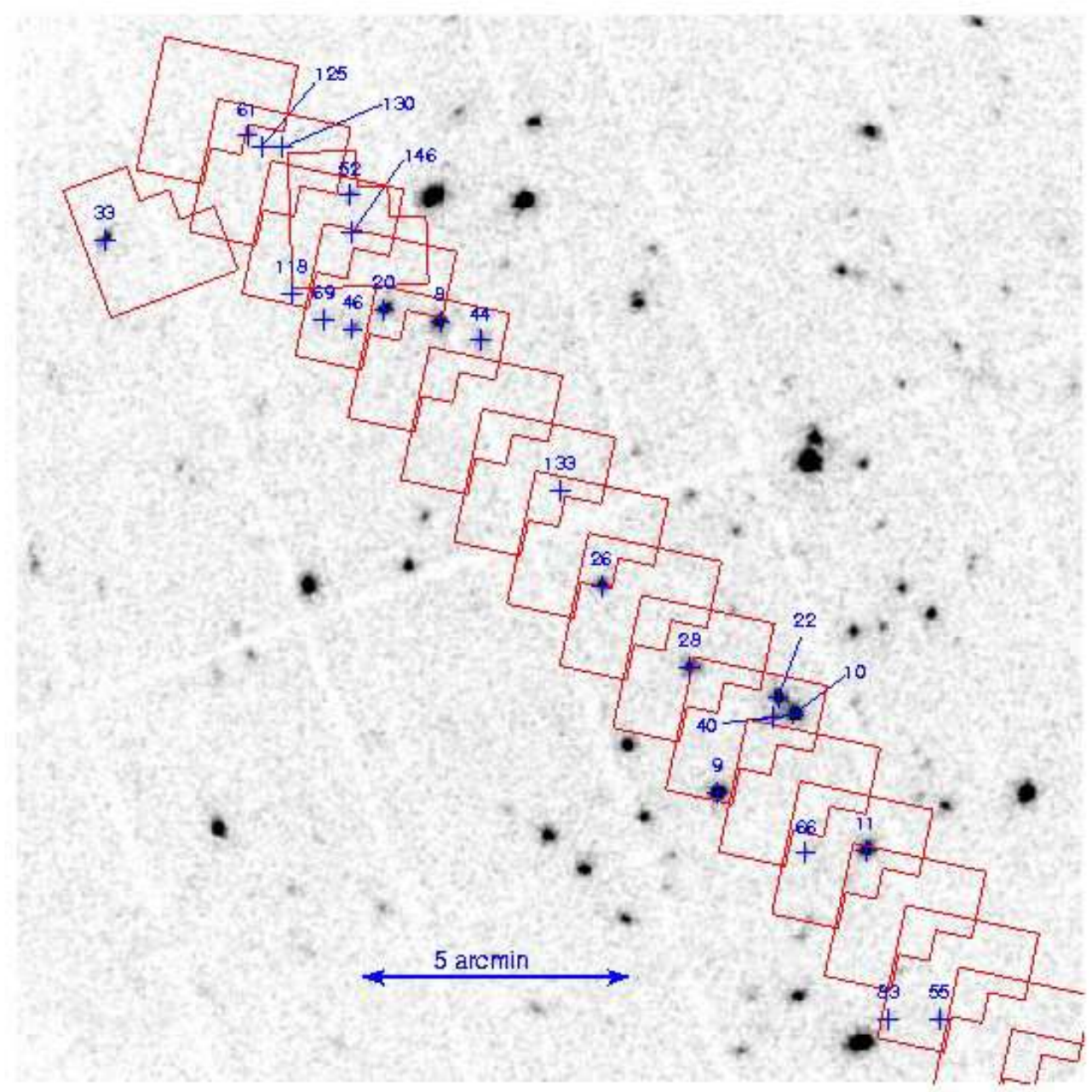

Fig. 1. - The WFPC2 fields of view are overlaid on the full band (0.5-10 keV) XMM-Newton image of the GWS. The underlying image has been smoothed with a $\sigma=2^{\prime \prime}$ Gaussian. The positions of the X-ray sources in Table 2 are marked. 
TABLE 2

XMM-Newton Sources In the WFPC2-StriP

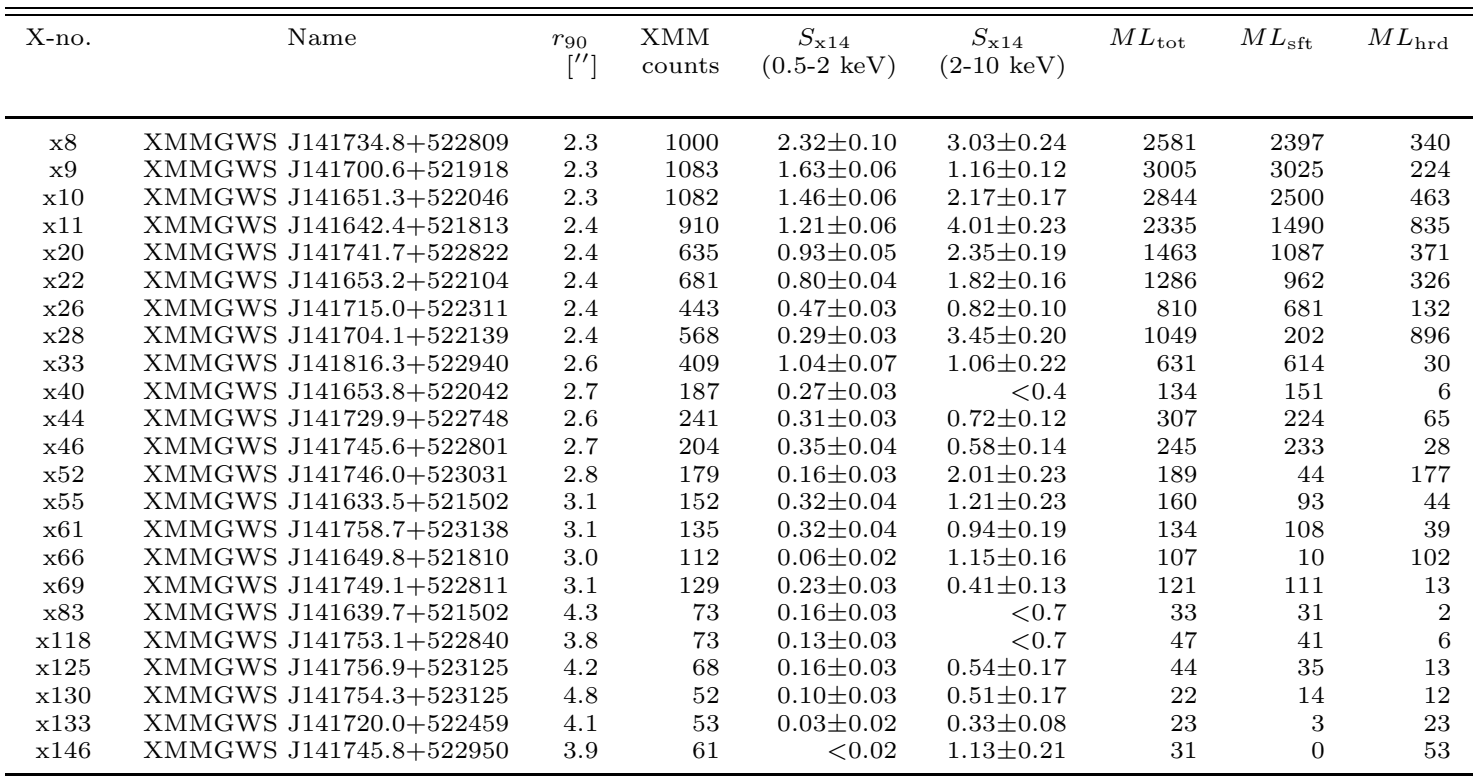

tions from the Chandra data are summarized in Table 3.

\subsection{X-ray Spectral Properties}

As a preview of the X-ray spectral properties of the detected sources, we plot a hardness ratio diagram in Fig. 2. The hardness ratio between the $2-4.5 \mathrm{keV}$ and $4.5-10 \mathrm{keV}$ is plotted against that between $0.5-2 \mathrm{keV}$ and $2-4.5 \mathrm{keV}$. The typical $1 \sigma$ errors of the hardness ratios are $0.05-0.2$; errors exceeding 0.4 are shown. We overplot grids showing the loci of absorbed power-law spectra for varying values of the photon index $\Gamma$ and intrinsic absorption $N_{\mathrm{H}}^{\mathrm{z}}$ for $\mathrm{z}=0.7$ and $\mathrm{z}=2$ respectively as described in the figure caption (Galactic $N_{\mathrm{H}}=1.310^{20} \mathrm{~cm}^{-2}$ has been included). Key optical/IR properties (Sect. 3) of the X-ray sources are indicated.

For bright sources, (XMM counts $\gtrsim 300$ ), Xray pulse-height spectral analysis gives further constraints on the spectral parameters. For the spectral analysis, we have used the data from the first observation in Table 1 (Rev/OBSID = 0113/0127921001), which contain mostly good intervals free from background flares. The extraction of the spectra has been made using the SAS

Table 3: Chandra Positions

\begin{tabular}{clll}
\hline XMM & \multicolumn{3}{c}{ Chandra } \\
X-nosition $(\mathrm{J} 2000)$ \\
$\mathrm{RA}\left({ }^{\circ}\right)$ & $\operatorname{DEC}\left({ }^{\circ}\right)$ & $r_{90}\left({ }^{\prime \prime}\right)$ \\
\hline x8 & 214.39514 & 52.46958 & 0.7 \\
x20 & 214.42441 & 52.47316 & 0.7 \\
x26 & 214.31275 & 52.38685 & 0.8 \\
x44 & 214.37476 & 52.46324 & 0.7 \\
x46 & 214.44027 & 52.46722 & 0.7 \\
x52 & 214.44140 & 52.50901 & 0.7 \\
x61 & 214.49547 & 52.52754 & 0.8 \\
x69 & 214.45498 & 52.46987 & 0.8 \\
x125 & 214.48674 & 52.52354 & 0.8 \\
x130 & 214.47583 & 52.52329 & 0.8 \\
x133 & 214.33364 & 52.41676 & 0.8 \\
x146 & 214.43923 & 52.49754 & 0.8 \\
\hline
\end{tabular}




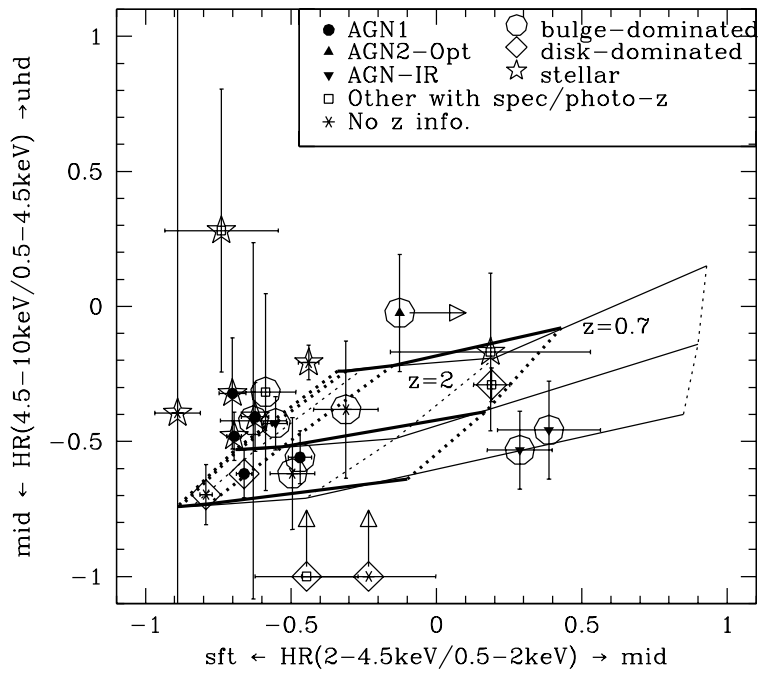

Fig. 2.- The X-ray color-color diagram of the Xray sources in GWS. The inner symbol shows the spectral classification (see Sect. 3; one narrowline Seyfert 1 has been included among AGN1s) and the outer symbol shows the morphological classifications as labeled. Error bars show $1 \sigma$ errors. The grid shows the location for absorbed power-law spectra at $\mathrm{z}=0.7$ (wider grid) and $\mathrm{z}=2$ (narrower grid, shown in thick lines). The solid lines correspond to photon indices of $\Gamma=1,2$, and 3 from top to bottom and the dotted lines to $\log N_{\mathrm{H}}=-\infty, 21.5,22.5$, and $23.5 \mathrm{~cm}^{-2}$ from left to right respectively. Objects $\mathrm{x} 44$ (X-ray source is off-nucleus) and x83 (Galactic star) are excluded from the plot. analysis package V5.4 and we have used the standard screening criteria with PATTERN $<5$ and PATTERN $<13$ for PN and MOS respectively as before. The calibration files used to generate the calibrated data products and response files for the spectral analysis (using the SAS task "epproc" and "emproc") were up to date as of Aug. 7, 2003. We extracted the source spectra using a circular region with a typical radius of $25^{\prime \prime}$, depending on the source count. The background spectra have been extracted using an annular region surrounding the source with inner and outer radii of $30^{\prime \prime}$ and $60^{\prime \prime}$ respectively. We have further jointly analyzed two co-added MOS spectra and the PN data.

For sources with $>600$ counts, we could reasonably constrain the parameters with a $\chi^{2}$ fit to the background subtracted spectra rebinned to have at least 20 counts per bin. For fainter sources, we have utilized the XSPEC implementation of the C-statistics (Cash 1979) to the spectra with much finer binnings. Because the $\mathrm{C}$-statistics cannot be applied to background-subracted data, the background and (source+background) spectra have been jointly fit. The background has been approximated by one or two power-laws and a broad Gaussian. This background model was then scaled by the source and background extraction areas and added to the source model. The result was then fitted to the source spectrum without background subtraction.

The source spectrum is fitted with a powerlaw, Galactic absorption (fixed at $N_{\mathrm{H}}=1.310^{20}$ $\mathrm{cm}^{-2}$ ), and an intrinsic absorption at the source redshift. For the sources without known redshifts, we have assumed a redshift for determining the value of $N_{\mathrm{H}}^{\mathrm{Z}}$. The results have been summarized in Table 4. See comments to this table for details.

\section{Optical Identifications and NIR Obser- vations}

This field has been targeted by a number of past and future survey projects in many wavelength, starting with the 28 contiguous HST WFPC observations (Groth et al. 1994). The main source of the optical data summarized in this paper comes from the HST MDS (Ratnatunga, Griffiths, \& Ostrander 1999) database and the DEEP survey. We have also made supplemental NIR spectroscopic observations for several of the X-ray sources. In- 
TABLE 4

Results of the X-RAY Spectral Analysis

\begin{tabular}{ccccccc}
\hline \hline X-No. & $z^{\mathrm{a}}$ & $\Gamma$ & $\begin{array}{c}N_{\mathrm{H}}^{\mathrm{z}} \\
10^{22} \mathrm{~cm}^{-2}\end{array}$ & $\begin{array}{c}S_{\mathrm{x} 14^{\mathrm{b}}} \\
\mathrm{erg} \mathrm{s}^{-1} \mathrm{~cm}^{-2}\end{array}$ & $\begin{array}{c}\log L_{\mathrm{x}} \mathrm{c} \\
\mathrm{erg} \mathrm{s}^{-1}\end{array}$ & $\begin{array}{c}\chi^{2} / \nu^{\mathrm{d}} \\
\text { or Prob.(\%) }\end{array}$ \\
\hline $\mathrm{x} 8$ & 1.223 & $2.2(2.0 ; 2.5)$ & $<1.3$ & 1.6 & 44.2 & $22 . / 38$ \\
$\mathrm{x} 9$ & $0.5^{*}$ & $2.5(2.3 ; 2.9)$ & $<0.07$ & 1.0 & $\ldots$ & $20 . / 47$ \\
$\mathrm{x} 10$ & 0.801 & $2.2(2.0 ; 2.8)$ & $<0.3$ & 1.4 & 43.7 & $7 . / 29$ \\
x11 & $1^{*}$ & $1.7(1.4 ; 2.2)$ & $1.6(0.7 ; 2.5)$ & 3.5 & $\ldots$ & $16 . / 31$ \\
x20 & 1.148 & $2.0(1.6 ; 2.7)$ & $1.8(0.7 ; 3.3)$ & 1.9 & 44.2 & $9 . / 19$ \\
x22 & 0.983 & $1.4(1.2 ; 1.7)$ & $<0.5$ & 2.7 & 44.2 & $11 . / 20$ \\
x28 & $0.76 \mathrm{p}$ & $1.2(0.8 ; 1.7)$ & $3.0(1.7 ; 5.3)$ & 4.1 & 43.8 & 62. \\
\hline
\end{tabular}

${ }^{a}$ Objects without spectroscopic redshifts are marked with an asterisk and the assumed redshift for $N_{\mathrm{H}}$ calculation is shown. Photometric redshifts are marked with "p".

bSource flux in the $2-10 \mathrm{keV}$ band at the observer's frame, corrected for Galactic absorption.

${ }^{\mathrm{c}}$ The base 10 logarithm of the source luminosity in the $2-10 \mathrm{keV}$ band at the source rest frame corrected for Galactic and intrinsic absorption.

${ }^{\mathrm{d}}$ For the $\chi^{2}$ fitting results, $\chi^{2}$ and the degrees of freedom are shown. For fits using Cstatistics, we give the percentage of simulated spectra drawn from a Gaussian distribution centered on the best fit with sigma from the covariance matrix which gives C-values smaller than the observed. (Using the xspec command "goodness" with the "sim" option.)

frared $\left(K_{\mathrm{s}}\right)$ photometry of these sources from the EMIR-COSMOS project(Cristóbal-Hornillos et al. 2003) are also included. The basic results are summarized in Table 5.

\subsection{Optical Morphology}

Fig. 3 shows the HST WFPC2 F814W images (with the exception of $\mathrm{x} 8, \mathrm{x} 20$, and $\mathrm{x} 44$, where the F606W images are shown) with $r_{0.9}$ error circles for XMM and Chandra (where applicable) source positions. Because each WFPC2 image may have absolute astrometric error of $\sim 1^{\prime \prime}$, in a few cases, the X-ray sources in one WFPC2 FOV show systematic offsets from their counterparts. In those cases, we have shifted the WFPC2 image by matching the positions of one of the X-ray sources with its unambiguous counterpart in making overlays. In Fig. 3, the overlays for x8, x20, and $\mathrm{x} 44$ have been tuned using $\mathrm{x} 8$, those for $\mathrm{x} 61$, $\mathrm{x} 125$, and $\mathrm{x} 130$ using $\mathrm{x} 61$, those for $\mathrm{x} 52$, x146, and x118 using $\mathrm{x} 146$, and those for $\mathrm{x} 69$, $\mathrm{x} 46$ and x20 using $x 69$. In any case, a translational shift without a rotation gives a satisfactory correction, where all the unambiguous counterparts (including Chandra sources without XMM detections) are within the Chandra error circles. Note that the drawn error circles $\left(r_{0.9}\right)$ may be an overestimate in these cases.

All of the X-ray source counterparts have an entry in the MDS database ${ }^{6}$, where the results of the disk-bulge decomposition are provided. The entries from the MDS database, including the MDSID, magnitudes in F814W (or $I_{814}$ ) and F606W (or $\left.V_{606}\right)$ filters, and the bulge-to-total brightness ratios $(\mathrm{B} / \mathrm{T})$ (or other morphological classifications) have been shown in Table 5 in columns (2),(3),(4), and (5) respectively. The first and second entries in column (5) (separated by a comma) are for the F606W and F814W bands respectively. If an entry in column (5) shows a number between 0 and 1 , it means that the host galaxy has been deconvolved with an exponential disk+bulge model and the bulge-to-total light ratio is shown. Other possible entries in this column are "B" (pure bulge), "D" (pure disk), "S" (unresolved point source), and "G" (galaxy; emission is extended, but could not discriminate between the exponential disk and bulge models).

\footnotetext{
${ }^{6} \mathrm{http}: / /$ archive.stsci.edu/mds
} 
TABLE 5

Optical-Infrared Morphological and Spectroscopic Properties

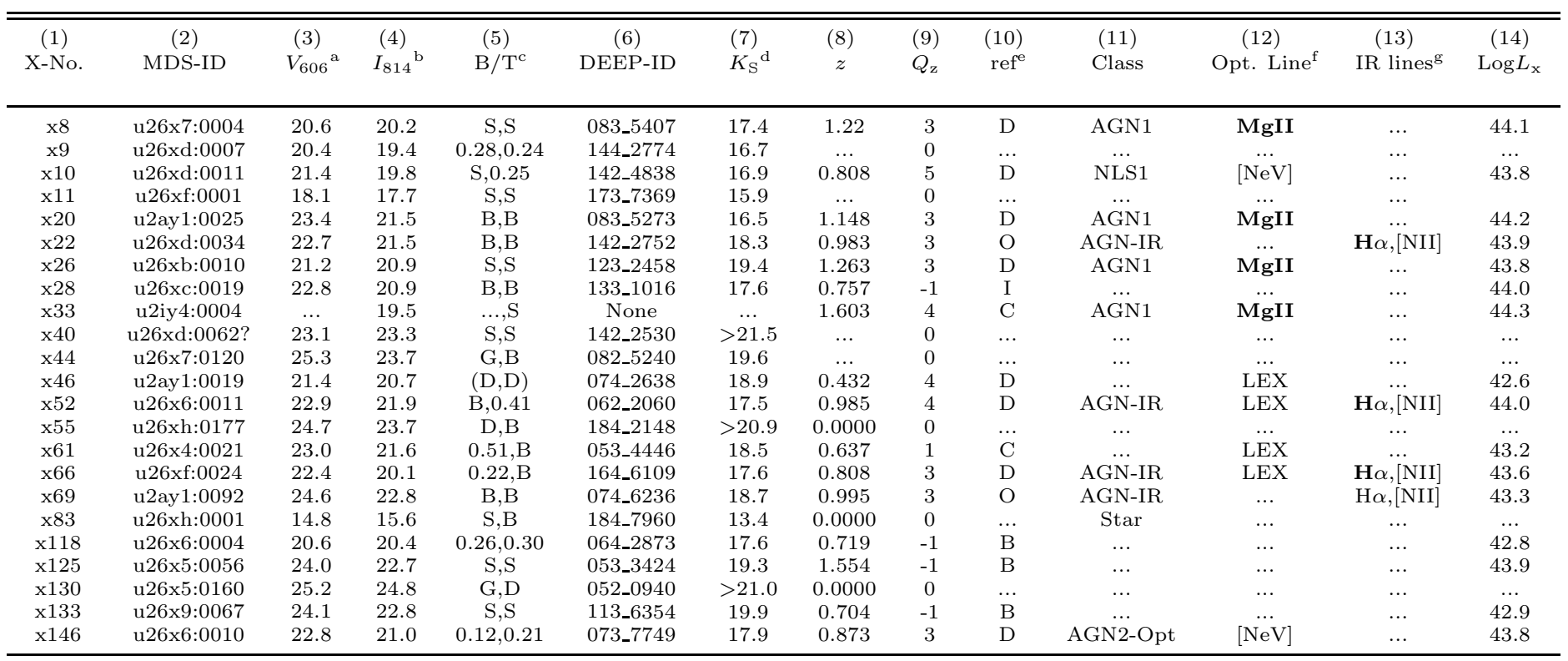

${ }^{a}$ Errors are smaller than 0.1 except (errors in parenthesis) x44 (0.1), x55 (0.2) and x130 (0.5).

${ }^{\mathrm{b}}$ Errors are smaller than 0.1 except for x55 (0.2) and x130 (0.2).

${ }^{\mathrm{c}}$ Bulge to total ratio if applicable, or the morphological class (B:pure bulge, D:pure disk, S:stellar, G:galaxy) for the $V_{606}$ band followed by that of the $I_{814}$ band.

${ }^{\mathrm{d}}$ Errors are smaller than 0.1 except for $\mathrm{x} 26(0.1), \mathrm{x} 44(0.2), \mathrm{x} 125(0.2)$ and $\mathrm{x} 133(0.1)$. Lower limit magnitudes correspond to the $3 \sigma$ detection limit.

${ }^{\text {e}}$ Redshift reference-D: DEEP C:CFRS B: Brunner (1999) I: Im et al. (2002) O: Subaru OHS (this work)

${ }^{\mathrm{f}}$ Only key features are shown. Broad lines are indicated by boldface. Those with only low-excitation narrow emission lines are labeled as LEX.

${ }^{\mathrm{g}}$ Broad lines are indicated by boldface. 


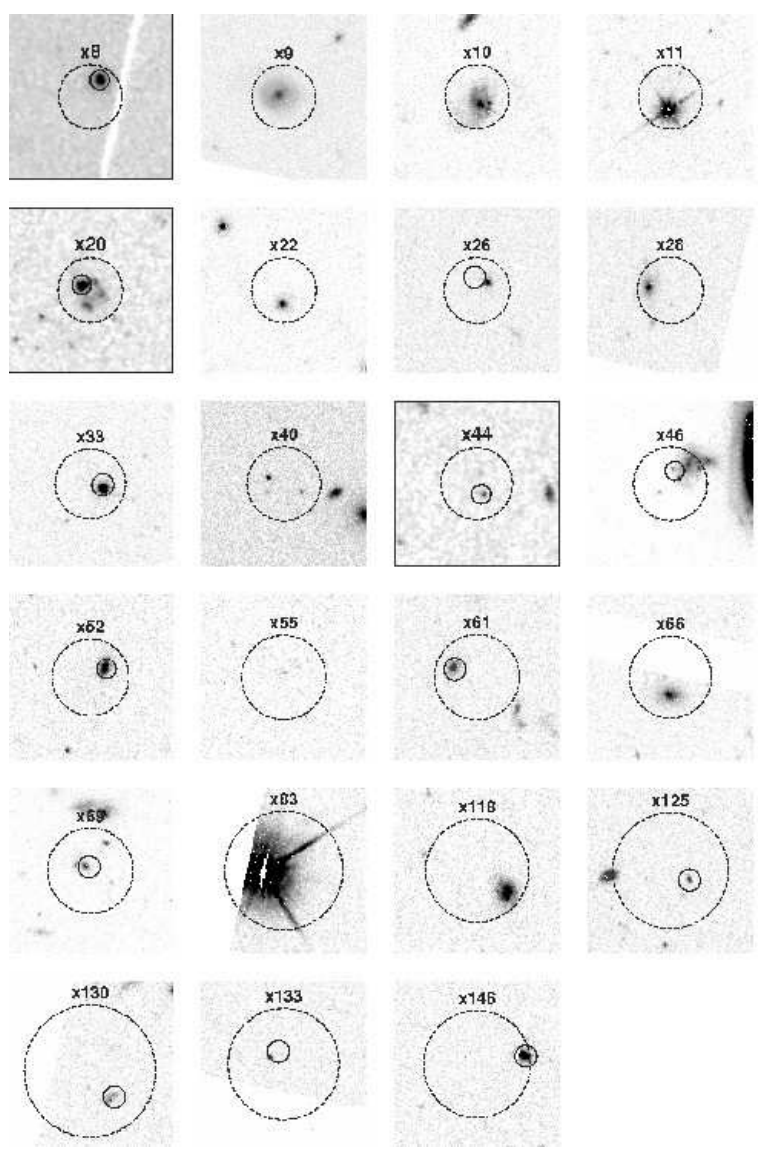

Fig. 3.- Postage stamp images from the HST WFPC2 images of the XMM-Newton sources in Table 2 are shown. Each image has a dimension of $12^{\prime \prime} \times 12^{\prime \prime}$. North is up and east is left. Large dashed circles and small solid circles are the $90 \%$ error circles of XMM-Newton and Chandra sources (if they exist) respectively.

\subsection{Optical Spectroscopic Classification}

Optical spectra are available for 8 of our Xray sources through the DEEP survey ${ }^{7}$ and two from the CFRS. Key AGN classification emission lines are shown in column (12) of Table 5. Broad permitted lines are indicated in boldface.

In summary, four X-ray sources show broad $\mathrm{Mg}$ II emission lines and we classify these as type 1 Seyfert/QSOs. They are identified with "AGN1" in column (12) of Table 5. Other objects with optical spectra show only narrow permitted and/or forbidden lines. Two of these show $[\mathrm{NeV}]$ emission, which is an unambiguous indicator of an AGN activity. One of these (x10) is a narrow-line Seyfert 1 galaxy ("NLS1" in column [11]) as discussed in Sect. 4.3. We tentatively classify the other object (x146) as a type 2 AGN and classify it as "AGN2Opt".

The other four objects do not show signs of AGN activity in their optical spectra in that they show neither broad permitted lines nor high excitation lines. These are classified as "LEX" (lowexcitation emission line objects) in column (12).

\subsection{Subaru CISCO/OHS NIR Spectroscopy}

We have made NIR spectroscopic observations for a subset of the X-ray sources with no optical spectroscopic indication of an AGN activity. These include objects with optical spectra which show only low excitation lines as well as those without optical spectra. Observations were made using the CISCO spectrometer with the $\mathrm{OH}$ suppressor (CISCO/OHS) (Motohara et al. 2002; Iwamuro et al. 2001) attached to the Nasmyth focus of the $8.2 \mathrm{~m}$ Subaru Telescope on March 22-24, 2003. The JH-Grism with a $0{ }^{\prime \prime} 5$ slit width was used. The spectral resolution of this configuration is $\lambda / \Delta \lambda=400$.

The primary purpose of the NIR spectroscopy was to detect the redshifted $\mathrm{H} \alpha \lambda 6563+[\mathrm{NII}]$ $\lambda \lambda 6548,6583$ complex and investigate the AGN nature of these X-ray sources. The ratio of the strengths of the [NII] doublet and $\mathrm{H} \alpha$ is a good discriminator between the AGN and starburst origins of the ionizing radiation. Typical Seyfert 2

\footnotetext{
${ }^{7}$ The spectra obtained by the DEEP team are publicly available and in the DEEP Public Database (http://saci.ucolick.org/verdi/public/index.html). Thus they have not been reproduced here.
} 
galaxies have $\log ([\mathrm{NII}] \lambda 6583 / \mathrm{H} \alpha)>-0.25$ (Veilleux \& Osterbrock 1987). We also intended to search for broad components in $\mathrm{H} \alpha$. Since a dust cloud is more transparent to redder light, moderately obscured AGN activities are much more visible in the longer wavelength lines such as $\mathrm{H} \alpha$ rather than shorter wavelength lines such as $\mathrm{H} \beta$ or $\mathrm{Mg}$ II. In fact, there have been a number of reported cases where X-ray source counterparts show broad $\mathrm{H} \alpha$ lines, even though they only show narrow lines in the shorter wavelength optical spectra (Akiyama, Ueda, \& Ohta 2002; Schmidt et al. 1998).

We did not include those with $\mathrm{z}<0.7$ ( $\mathrm{x} 46$, $\mathrm{x} 61$ ) in our NIR spectroscopy targets, because the $\mathrm{H} \alpha+[\mathrm{NII}]$ line complex does not fall within the wavelength range observable by CISCO/OHS JHgrism. Also in order to make spectroscopic observation feasible within a reasonable exposure time, we limited our spectroscopic observation to those with an estimated $\mathrm{H}$-band magitude of $H<21$ (Iwamuro et al. 2001), where $\mathrm{H}$ band magnitudes were estimated by $H \approx I_{814}-1.4$ (Barger, Cowie, Mushotzky, \& Richards 2001). Five from the 23 XMM-Newton sources were observed during our observing run.

The resulting spectra, reduced following the procedure by Iwamuro et al. (2001), are shown in Fig 4. Preliminary flux calibrations have been made using a nearby standard star observed immediately after the target.

We have detected the $\mathrm{H} \alpha+[\mathrm{NII}]$ complex in 4 of the 5 observed X-ray sources. In all four cases, the spectra suggest a possible broad $\mathrm{H} \alpha$ component (FWHM $\sim 2000 \mathrm{~km} \mathrm{~s}^{-1}$ ) and/or strong [NII] lines. This is indicated in column (13) of Table 5 and the sources are classified as "AGN-IR" in column (11).

Because of the weak signal-to-noise ratio and difficulty in determining the background, careful analysis is required to make any quantitative conclusions. This will be the topic of a separate paper that will describe the details of the observations, data reduction, and quantitative analyses for the Subaru CISCO-OHS spectra of the sources shown in this paper and a few additional sources observed during the observation run.

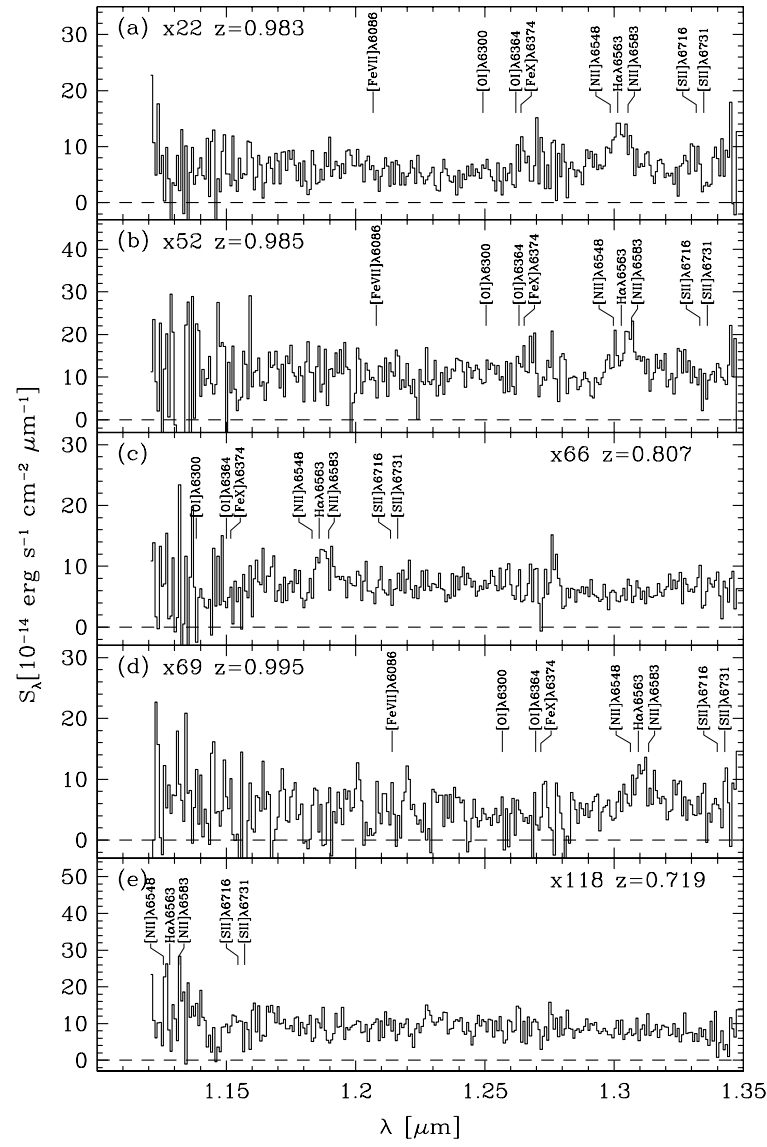

Fig. 4.- Near-infrared (NIR) spectra of the XMM-Newton sources observed with CISCO-OHS on the Subaru Telescope. Redshifted positions of common QSO emission lines from Wilkes (2000) are marked. 


\subsection{Infrared $\left(K_{\mathrm{S}}\right)$ Photometry}

The $K_{\mathrm{s}}$ photometry was provided by the EMIRCOSMOS project (Guzmán 2003; Balcells 2003). The GWS was imaged with the near-infrared camera INGRID on the William Herschel Telescope in the Spanish Observatorio del Roque de los Muchachos during two runs in 2000 April and 2001 June. These data are fully described in CristóbalHornillos et al. (2003). Atmospheric conditions were photometric, with seeing ranging from $0.6^{\prime \prime}$ to $0.75^{\prime \prime}$ FWHM in 2000 April, and $0.60^{\prime \prime}-1.30^{\prime \prime}$ FWHM in 2001 June. Source extraction and photometry of $K_{\mathrm{s}}$-band data was done using SExtractor (Bertin \& Arnouts 1996). Zeropoint uncertainty is 0.03 mag. SExtractor BEST magnitudes are provided with errors including photon noise. The resulting $K_{\mathrm{s}}$ magnitudes are listed under column (7) of Table 5.

\subsection{Redshifts and X-ray Luminosities}

Redshifts, redshift quality flag and the reference source of the redshift are shown in columns (8), (9), and (10) of Table 5. The meanings of the redshift quality flags are: -1 : photometric redshift only, 0: no redshift information, 1-5: as defined by the DEEP project (Lowenthal et al. 1997) or the CFRS (Lilly, Hammer, Le Fevre, \& Crampton 1995) confidence class if the redshift source is CFRS. The redshift of x22 has been solely determined by the $\mathrm{H} \alpha+[\mathrm{NII}]$ complex from the Subaru OHS data. Since this feature is unambiguous, it has been assigned the quality flag of 3 .

For those with redshift information, we have calculated the X-ray luminosity of the source and listed the results in column (13). The luminosities have been calculated from the $2-10 \mathrm{keV}$ rest-frame fluxes given in Table 2 and the K-corrections have been made using the spectral assumptions described in Sect. 2. The luminosities have been corrected for possible intrinsic absorption using the same spectral assumption. Because of the difference in methods and exact time selections, the luminosities listed here can be slightly different from those in the full spectral analysis listed in Table 4.

\section{Results and Discussion}

\subsection{X-ray Source Population}

The 23 sources detected in our $80 \mathrm{ks}$ XMMNewton observation of the GWS are representative of the X-ray sources that contribute most to the "Cosmic X-ray Background" and many of them represent the regime which marks the peak of accretion onto SMBHs in centers of galaxies.

A dominant population in this field consists of AGNs with $\log L_{\mathrm{x}} \sim 44$ at $z \sim 1$. Only a few of them show signs of AGN activity in their optical spectra. Subaru OHS NIR spectroscopy of four of the X-ray sources with no previous optical signature of AGNs revealed $\mathrm{H} \alpha+[\mathrm{NII}]$ emission lines showing hints of broad $\mathrm{H} \alpha$ and/or stronger narrow [NII] lines indicative of AGN activity.

The host galaxies of the X-ray sources tend to be bulge-dominated and four are extremelyred objects (EROs) (or Very Red Objects; VROs) $\left(I_{814}-K_{\mathrm{s}} \geq 4\right)$. Also one object (x130) has an upper limit $I_{814}-K_{\mathrm{s}}<4.8$, which is consistent with being an ERO. Fig. 5 shows $V_{606}-I_{814}$ and $I_{814}-K_{\mathrm{S}}$ colors of the X-ray sources in our sample as a function of redshift. We have excluded x33 (no $K_{\mathrm{s}}$ and $V_{606}$ photometry available),x46 (X-ray source is off-nucleus, see below), and x83 (Galactic star) from the plot. Those without redshift information are plotted left of $\mathrm{z}=0$. For reference, we plot K- and evolution-corrected galaxy colors for elliptical (labeled as E1 in Fig. 5), Sa and Sc galaxies from Poggianti (1997). Model E1 corresponds to Poggianti's model "E", which has a star-formation rate with e-folding time of 1 Gyr. We have neglected the difference of cosmological parameters used by us andPoggianti (1997), which corresponds to $\mathrm{a} \sim 10 \%$ difference in the age of the Universe. In plotting these color tracks, we have converted the $I$ and $V$ magnitudes to $I_{814}$ and $V_{606}$ using the formulae given by Cowie, Songaila, \& Barger (1999). We also plot the colors of an elliptical galaxy model, which we have calculated for our filters and cosmology from the evolving SEDs by Kodama \& Arimoto (1997) (labeled as E2). The plotted model is for a passively evolving galaxy after a short burst at the formation epoch of $z_{\mathrm{f}}=4.62$ and $L=0.1 L_{*}$ at $z=0$. The difference between $K$ and $K_{\mathrm{s}}$ magnitudes have been neglected, following Cristóbal-Hornillos et al. (2003). 


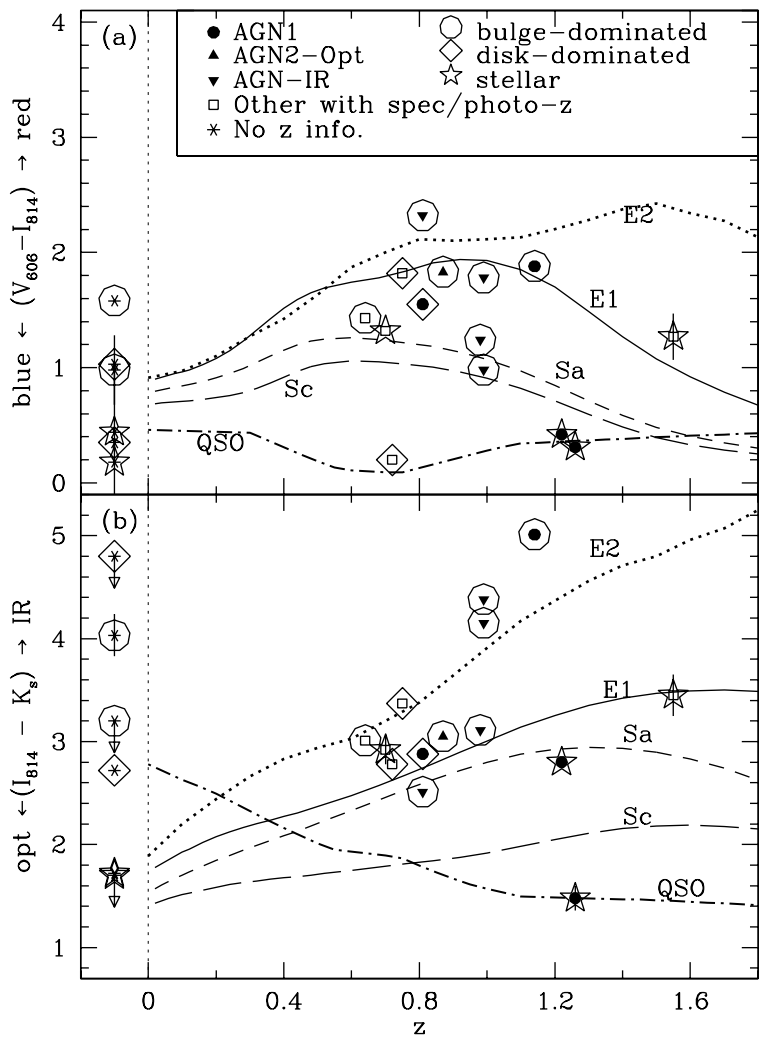

Fig. 5.- The $V_{606}-I_{814}$ (upper panel) and $I_{814}-K_{\mathrm{s}}$ colors of the $\mathrm{X}$-ray sources plotted as a function of redshift. Those without redshift information are plotted left of zero. The meanings of the symbols are the same as those in Fig. 2. One sigma error bars are shown if $\sigma \geq 0.1$. The solid, short-dashed, long-dashed curves are galaxy colors as functions of $z$, based on model spectra for Elliptical (E1), Sa, and Sc galaxies after K- and evolution corrections given by Poggianti (1997). The thick dotted line show the colors of a passively evolved elliptical model by Kodama \& Arimoto (1997). The dot-dashed lines are the colors calculated from the mean radio-quiet QSO spectrum by Elvis et al. (1994).
Fig. 5 shows that the $I_{814}-K_{\mathrm{S}}$ color of the $\mathrm{X}$-ray sources with bulge-dominated hosts indeed traces that of elliptical galaxies and, in particular, those of EROs with redshifts are roughly consistent with the passively evolving elliptical model (E2). The $V_{606}-I_{814}$ color, which is more sensitive to the contaminations from star formation and AGN activities, shows a scatter towards bluer colors from the elliptical regime. The reddest one (x20), with $I_{814}-K_{\mathrm{s}}=5.0$ may be contributed by a dust enshrouded AGN or a starburst. Pointlike sources tend to be distributed towards bluer (QSO) colors, although the scatter is large. The scatter may be contributed to by unresolved host galaxies, intrinsic scatter in QSO colors and/or reddening by intrinsic dust absorption.

\subsection{Bulge Mass and X-ray Luminosity}

The HST imaging of this field has allowed us to find a significant population of X-ray sources at $z \sim 1$ whose counterparts have a resolved bulge component, either as part of a disk+bulge structure or a pure bulge. In view of the relationship found between bulge mass and the central blackhole mass in nearby galaxies (Magorrian et al. 1998; Merritt \& Ferrarese 2001), it is interesting to make a first-order estimation of blackhole mass $\left(M_{\bullet}\right)$ from the bulge-component of the host galaxy and compare it with the X-ray luminosity. In the rough estimation below, we take the approach of Aller \& Richstone (2002) by first converting the bulge luminosity to the central velocity dispersion $(\sigma)$ of the bulge stellar component using an empirical relation. Then we use the tight $\sigma-M_{\bullet}$ (Merritt \& Ferrarese 2001; Tremaine et al. 2002) correlation to obtain the estimated blackhole mass. We use the F814W K-correction for the E galaxy in Fig. 18(d) of Fukugita, Shimasaku, \& Ichikawa (1995) to calculated the F814W absolute magnitude $\left(M_{\mathrm{F} 814 \mathrm{~W}}\right)$. We also assume an earlytype galaxy color of

$$
M_{\mathrm{B}_{\mathrm{T}}}-M_{\mathrm{F} 814 \mathrm{~W}}=2.1
$$

(Fukugita, Shimasaku, \& Ichikawa 1995; Gonzalez et al. 2000). We then use the relations,

$$
\begin{array}{r}
-M_{\mathrm{B}_{\mathrm{T}}}+5 \log h_{70}=20.5+7.7(\log \sigma-2.3), \\
M_{\bullet} / M_{\odot}=1.4810^{8}(\sigma / 200)^{4.65},
\end{array}
$$

(Gonzalez et al. 2000; Merritt \& Ferrarese 2001) to obtain the estimated blackhole mass. Because 
the $\mathrm{F} 814 \mathrm{~W}$ band corresponds to the $B$ band at the source rest frame of $z \sim 1$, the combination of the K-correction and the magnitude conversion to $M_{\mathrm{B}_{\mathrm{T}}}$ is insensitive to the assumed galaxy spectral energy distribution.

Fig. 6 shows the estimated $M_{\bullet}$ versus $L_{\mathrm{x}}$ (absorption corrected, see Sect. 3.5) for $10 \mathrm{X}$-ray sources in the sample which have a resolved bulge component in the HST WFPC2 F814W image (MDS).

It is interesting to estimate the Eddington ratio $L_{\mathrm{bol}} / L_{\mathrm{Edd}}$ for these X-ray sources, where $L_{\mathrm{bol}}$ is the bolometric luminosity of the AGN and $L_{\mathrm{Edd}}$ is the Eddington luminosity corresponding to the blackhole mass. Writing $L_{\mathrm{bol}}=b L_{\mathrm{x}}$, where $b \approx$ 25 (Elvis et al. 1994), we overplot three lines showing $\left(L_{\mathrm{bol}} / L_{\mathrm{Edd}}\right)(25 / b)=10^{-1}, 10^{-2}$, and $10^{-3}$ in Fig. 6. Fig. 6 shows that the estimated blackhole masses for the 10 AGNs range from $10^{7}-10^{10} M_{\odot}$ and the Eddington ratios from $0.3 \%-10 \%$.

These results have interesting implications on how AGN evolve and the current stage of evolution for the AGN represented here $(z \sim 1$, $\left.\log L_{\mathrm{x}} \sim 44\right)$. This is indeed a characteristic redshift and luminosity marking the peak of the accretion history of the universe. The result that these AGN are typically radiating at a few percent may have important implications on the accretion history and formation of SMBHs. If this is a typical Eddington ratio throughout the AGN phase of these objects, the growth of the blackhole occurs on a timescale of $\sim t_{\mathrm{s}} /\left(L_{\mathrm{bol}} / L_{\mathrm{Edd}}\right) \sim$ a few $\times 10^{9} \mathrm{yrs}$, where $t_{\mathrm{s}} \sim 510^{7}\left(\frac{\epsilon}{1-\epsilon}\right)\left(\frac{0.1}{1-0.1}\right)^{-1} \mathrm{yrs}$ is the Salpeter timescale, that is the timescale at which the mass of an object accreting at the Eddington Luminosity grows by a factor of $e$ (for a radiative efficiency $\epsilon \sim 0.1$ of a standard accretion disk model). This scenario has difficulty in that the timescale of a few $10^{9}$ yrs may be too long, while the number density of AGN at $\log L_{\mathrm{x}} \lesssim 44$ decreases beyond $z \sim 1$ (Ueda, Akiyama, Ohta, \& Miyaji 2003). Alternatively, it is possible that these AGN have gone through a brief luminous phase in the past with near-Eddington accretion rates. These may have been observed as more luminous QSOs $\left(\log L_{\mathrm{x}} \gtrsim 45\right)$ at $z>2$. Yet another more exotic possibility is that they are just accreting with a low radiative efficiency $(\epsilon<<0.1)$, allowing much less time for the SMBH to grow.

We note, however, that there are a number of

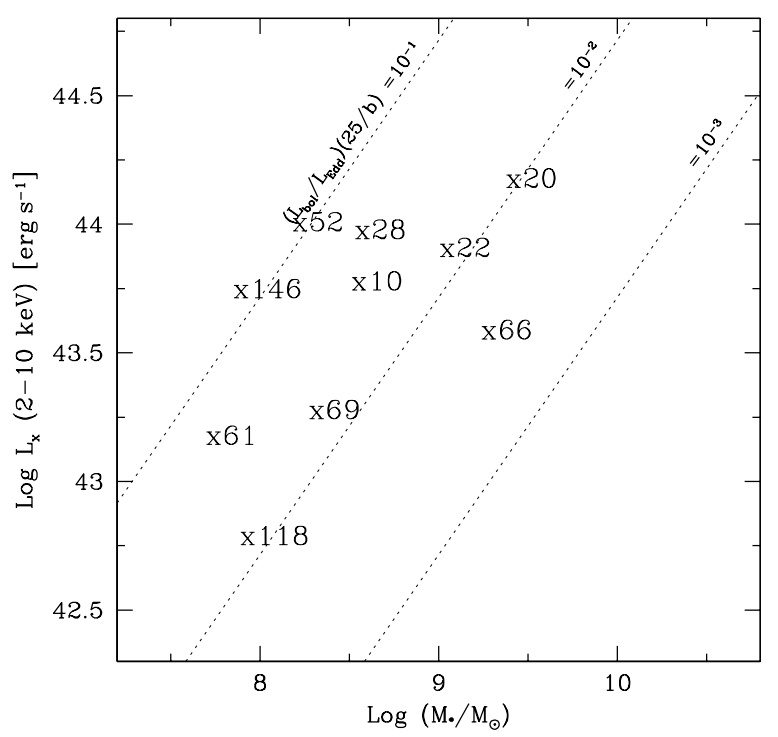

Fig. 6. - The X-ray luminosity (absorptioncorrected) plotted as a function of the central blackhole mass $\left(M_{\bullet}\right)$ estimated from the bulge luminosity (see text) for the $10 \mathrm{X}$-ray sources in the sample with resolved bulge components. The data points are shown with X-name labels. Three lines correspond to $\left(L_{\mathrm{bol}} / L_{\mathrm{Edd}}\right)(25 / b)=10^{-1}, 10^{-2}$, and $10^{-3}$, where $L_{\mathrm{bol}}=b L_{\mathrm{x}}$ is the bolometric luminosity of the AGN component and $L_{\mathrm{Edd}}$ is the Eddington luminosity corresponding to $M_{\bullet}$. 
caveats in interpreting these results and drawing conclusions relating the X-ray AGN evolution and growth of the SMBH. Firstly, the current MDS database shows the analysis for stellar (pointlike) images or galaxies (pure bulge, pure disk or bulge+disk decomposition), but only limited analysis (Sarajedini, Green, Griffiths, \& Ratnatunga 1999) has been completed for decomposing pointlike (stellar) nuclei from the host galaxy (stellar+bulge+disk, stellar+bulge or stellar+disk). Thus we select against those with strong AGN components (or with large $\left(L_{\mathrm{bol}} / L_{\mathrm{Edd}}\right)$, which are likely to be listed as "stellar" in the MDS database or the bulge luminosity in the database may be contaminated by the central AGN component. This situation should be improved in the future, where the HST images are analyzed with pointlike nucleus+host galaxy decomposition. Extending this study to other deep fields with X-ray and HST (WFPC2 as well as ACS) data, including the Extended Chandra Deep Field-South (E-CDFS) and the COSMOS field, will be a next logical step. Secondly, a much more fundamental limitation is that we have assumed the local relations in Eq. 2 are still valid at $z \sim 1$. This assumption is not guaranteed to be valid.

\subsection{Comments on Selected Individual Ob- jects}

x8: This is a typical type 1 QSO at $z=1.22$ with a broad Mg II line.

x10: The DEEP optical spectrum shows many narrow emission lines including high-excitation lines like $[\mathrm{NeV}] \lambda \lambda 3346,3426$, which are unambiguous indicators of AGN activity. Permitted lines ( $\mathrm{Mg} \mathrm{II}, \mathrm{H} \beta$ ) are also narrow. Since $\mathrm{H} \beta / /[\mathrm{OIII}] \lambda 5007 \approx 0.7>1 / 3$, these lines are not dominated by a Seyfert 2 . It is either a Seyfert 2 whose emission lines are heavily contaminated by starburst activity or a narrow-line Seyfert 1 galaxy (NLS1) (Osterbrock \& Pogge 1985), where $\mathrm{H} \beta$ is contributed to from the (narrow end of the) broad line region. Because our X-ray spectral analysis shows no X-ray absorption $\left(N_{\mathrm{H}}<10^{21.5} \mathrm{~cm}^{-2}\right.$; see Table 4$)$, the NLS1 interpretation is more plausible.

x11: The optical counterpart has a very bright stellar (point-like) morphology and there is no optical spectrum available for this source to discriminate between a galactic star and a QSO. However, its X-ray spectrum is inconsistent with a thermal plasma, having a significant residual in the soft part. Also its Xray to optical flux ratio $\log \left(f_{\mathrm{X}} / f_{\mathrm{R}}\right) \approx-0.7$ ( $f_{\mathrm{x}}$ is measured in $2-10 \mathrm{keV}$ ) is well within the AGN regime (roughly between -1 and 1; see e.g. Hornschemeier et al. 2001). Thus it is most likely to be a QSO.

x20: Thanks to the Chandra position, we can identify the X-ray source with the brightest bulge-dominated galaxy at $z=1.148$, among four candidates apparently interacting with one another indicated by tidal bridges (See Fig. 3). It is an interesting case where galaxy interactions are possibly feeding the AGN activity at this early stage of the universe. It has a QSO luminosity $\left(\log L_{\mathrm{x}}=44.2\right)$, but the optical image is dominated by bulge component of the host galaxy. The DEEP spectrum shows a broad $\mathrm{Mg}$ II line. The X-ray spectrum shows an absorption of $\log N_{\mathrm{H}} \sim 22.3$. This is an example of optical type-1 X-ray type 2 AGN. This is also an ERO $\left(I_{814}-K_{\mathrm{s}}=5.0\right)$ and a sub-mm source detected in a deep SCUBA survey (Waskett et al. 2003).

x22: No previous optical/IR spectroscopic observations existed for this source. Our Subaru OHS/CISCO observation detected $\mathrm{H} \alpha$ and $[N I I] \lambda \lambda$ 6548,6583 emission lines, giving $\mathrm{z}=0.983$. Based on the sign of broad $\mathrm{H} \alpha$ and strong $N I I$, high luminosity $\log L_{\mathrm{x}} \sim$ 44.2. While we mark it as an AGN-IR, it may well be a type 1 AGN.

x28: This source is the most conspicuous hard Xray source in the field with an intrinsic absorption of $\log N_{\mathrm{H}} \sim 22.5\left[\mathrm{~cm}^{-2}\right]$. It is a bulge-dominated galaxy with no optical/IR spectroscopy and a photometric redshift of $z=0.76$ (Im et al. 2002) based on its V-I color alone. This is most likely a Seyfert 2 based on the X-ray properties.

x46: The position of the source has been determined with Chandra and the relative alignment of the WFPC2 image and Xray sources has been achieved the three 
other CXO sources in the field. The Xray source counterpart is identified with a hot spot just off the patchy irregular starforming galaxy DEEP gss 074_2638 (MDS $\mathrm{u} 2 \mathrm{ay1}$ :0019) at $\mathrm{z}=0.432$. If we assume that the X-ray source is at the same redshift as this irregular galaxy, the luminosity would be $\log L_{\mathrm{x}} \approx 42.8$. This is too luminous for an ultraluminous X-ray source (ULX). We also note that there is a nearby edge-on disk galaxy (DEEP gss 074_2237, $\mathrm{z}=0.156$ ). The X-ray source is $4^{\prime \prime}$ away (projected distance of $10 \mathrm{kpc}$ ) from its nucleus and located towards the direction perpendicular to the disk. Therefore the X-ray source could be a ULX associated with the halo of the galaxy. However, even if the X-ray source was at the redshift of this disk galaxy, its luminosity would still be $\log L_{\mathrm{x}}=41.8$, again well above the ULX regime. One possibility is that this irregular galaxy is undergoing a merging process and the X-ray source is at the nucleus of one of the merging galaxies, or it may simply be a background QSO.

x52: The DEEP spectrum show no broad lines and the location of MgII shows only absorption. Other features include [OII] $\lambda 3727$ and $[\mathrm{NeIII}] \lambda 3869$. The presence of $[\mathrm{NeV}] \lambda 3426$ is suggested but uncertain. We observed this object with Subaru OHS and found a moderately broad $\mathrm{H} \alpha\left(\mathrm{FWHM} \sim 2000 \mathrm{~km} \mathrm{~s}^{-1}\right)$ and a strong [NII] doublet. (Fig. 4). The fact that there is no broad MgII line but a moderately broad $\mathrm{H} \alpha$ suggests that the AGN is obscured by a dust cloud. This is consistent with the hard color of this object, with the second largest $\mathrm{HR}(2-4.5 \mathrm{keV} / 0.5-2 \mathrm{keV})$ in the sample. See Fig. 2. This is an ERO $\left(I_{814}-K_{\mathrm{s}}=4.4\right)$.

x66: We find no indication of an AGN in the DEEP spectrum. Like x52, our Subaru OHS observation revealed possible AGN activity through the detection of a moderately broad $H \alpha\left(\right.$ FWHM $\sim 2000 \mathrm{~km} \mathrm{~s}^{-1}$ ) line and a strong [NII] doublet. This is an obscured AGN similar to $x 52$ and has a hardest $\operatorname{HR}(2-$ $4.5 \mathrm{keV} / 0.5-2 \mathrm{keV})$. There is also a hint of $[\mathrm{NeIII}] \lambda 3869$, a line which is often stronger in AGN than starforming galaxies. x69: A spectroscopic redshift of $z=0.995$ has been determined by our Subaru observation in close agreement with the photometric redshift determined by Brunner (1999) of $z_{\mathrm{ph}}=0.935$. The Subaru OHS spectrum seems to indicate either strong $[\mathrm{NII}] \lambda 6583$ or broad $\mathrm{H} \alpha$ suggestive of AGN activity. This is an $\operatorname{ERO}\left(I_{814}-K_{\mathrm{s}}=4.1\right)$.

x83: Because of the low X-ray to optical flux ratio $\left(\log \left(f_{\mathrm{x}} / f_{\mathrm{R}}\right) \approx-3\right)$ for this bright optical object $(\mathrm{F} 606 \mathrm{~W}=14.8)$, this is certainly a Galactic star.

x125: Although the MDS database shows that it is a point source, its color is consistent with an elliptical galaxy at the photometric redshift of $z_{\mathrm{ph}}=1.55$ (Brunner 1999). The optical counterpart is probably dominated by the host galaxy.

$\mathbf{x 1 4 6}$ : This object is detected only in the hard (2$8 \mathrm{keV}) \mathrm{X}$-ray band. The DEEP optical spectrum clearly shows $[\mathrm{NeV}] \lambda 3426$ emission and strong $[\mathrm{NeIII}] \lambda 3869$. This is a typical Seyfert 2 galaxy with absorbed X-ray spectrum.

\section{Summary}

1. We summarize the properties of X-ray sources in the GWS detected with XMMNewton and discuss their morphology, optical and NIR spectra.

2. Ten out of the $23 \mathrm{X}$-ray sources have spectroscopic information available in the optical band through the DEEP and CFRS projects. Four of them have broad emission lines and thus can be classified as type 1 AGN/QSOs. Two others have high excitation lines indicative of narrow line region of active galactic nuclei. Others do not reveal AGN signatures in their optical spectra.

3. We have obtained NIR spectra for five of the sources which did not reveal AGN signatures in their optical spectra using the CISCO/OHS spectrograph on the Subaru Telescope. In four of the five, we detect broad $\mathrm{H} \alpha$ and/or strong [NII] lines suggestive of AGN activity. 
4. The X-ray hardness ratios and spectroscopic analysis show that a major part of the observed X-ray sources consists of a mixture of absorbed and unabsorbed AGNs. One of these AGNs (x20) has an absorbed X-ray spectrum with a broad MgII emission line and is another example of an optical type 1/X-ray type 2 AGN.

5. The $V_{606}-I_{814}$ and $I_{814}-K_{\mathrm{s}}$ colors of the $\mathrm{X}$-ray sources with bulge-dominated hosts well trace those of evolving elliptical galaxies. Sources with stellar morphologies show bluer colors with some scatter, and consistent with AGN(QSO) with a host galaxy contamination and/or dust absorption.

6. Ten X-ray sources with redshift information have a resolved bulge. Based on a number of assumptions concerning the bulge luminosity-blackhole mass relation, we estimate that these AGN have luminosities ranging from $0.3-10 \%$ of the Eddington luminosity, suggesting that these massive blackholes have previously gone through massive accretion (maybe as luminous QSOs) or that their lifetimes are relatively long ( $\left.\gtrsim 10^{9} \mathrm{yrs}\right)$.

The authors are indebted to the efforts of the DEEP team for their observations, analysis, and archiving. This work has been supported by the NASA Grant NAG5-10875 to TM (Long-Term Space Astrophysics), NASA Grant NAG5-3651 to REG (XMM-Newton Mission Scientist support).

\section{REFERENCES}

Akiyama, M., Ueda, Y., \& Ohta, K. 2002, ApJ, 567,42

Alexander, D. M., Vignali, C., Bauer, F. E., Brandt, W. N., Hornschemeier, A. E., Garmire, G. P., \& Schneider, D. P. 2002, AJ, 123, 1149

Aller, M. C. \& Richstone, D. 2002, AJ, 124, 3035

Balcells, M. 2003, Revista Mexicana de Astronomía y Astrofísica, 16, 69

Baldi, A., Molendi, S., Comastri, A., Fiore, F., Matt, G., \& Vignali, C. 2002, ApJ, 564, 190
Barger, A. J., Cowie, L. L., Mushotzky, R. F., \& Richards, E. A. 2001, AJ, 121, 662

Beck-Winchatz, B. \& Anderson, F. 1999, AJ 117, 2582

Bertin, E. \& Arnouts, S. 1996, A\&AS, 117, 393

Brunner, B.J. 1999, PhD Thesis, Johns Hopkins University

Cash, W. 1979, ApJ, 228, 939

Cowie, L. L., Songaila, A., \& Barger, A. J. 1999, AJ, 118, 603

Cristóbal-Hornillos, D., Balcells, M., Prieto, M., Guzmán, R., Gallego, J., Cardiel, N., Serrano, Á., \& Pelló, R. 2003, ApJ, 595, 71

Dickey, J. M. \& Lockman, F. J. 1990, ARA\&A, 28,215

Elvis, M. et al. 1994, ApJS, 95, 1

Fukugita, M., Shimasaku, K., \& Ichikawa, T. 1995, PASP, 107, 945

Gonzalez, A. H., Williams, K. A., Bullock, J. S., Kolatt, T. S., \& Primack, J. R. 2000, ApJ, 528, 145

Guzmán, R. 2003,Revista Mexicana de Astronomía y Astrofísica, 16, 209

Groth, E. et al. 1994, BAAS 185, 5309

Hornschemeier, A. E. et al. 2001, ApJ, 554, 742

Hornschemeier, A. E. et al. 2003, AJ, 126, 575

Im, M. et al. 2002, ApJ, 571, 136

Iwamuro, F., Motohara, K., Maihara, T., Hata, R., \& Harashima, T. 2001, PASJ, 53, 355

Kodama, T. \& Arimoto, N. 1997, A\&A, 320, 41

Lehmann, I. et al. 2001, A\&A, 371, 833

Lilly, S. J., Hammer, F., Le Fevre, O., \& Crampton, D. 1995, ApJ, 455, 75

Lowenthal, J. D. et al. 1997, ApJ, 481, 673

Magorrian, J. et al. 1998, AJ, 115, 2285 
Merritt, D. \& Ferrarese, L. 2001, in The Central Kiloparsec of Starbursts and AGN, ed. J. H. Knapen et al. (San Francisco: ASP), 335

Miyaji, T., Griffiths, R. E. 2001 in Galaxy Clusters and the High Redshift Universe Observed in X-rays: proceedings of the XXIst Moriond Astrophysics Meeting, eds. D. Neumann et al. (CD-ROM)

Miyaji, T. \& Griffiths, R. E. 2002, ApJ, 564, L5

Miyaji, T., Griffiths, R. E., Lumb, D., Sarajedini, V., \& Siddiqui, H. 2003, Astronomische Nachrichten, 324, 24

Motohara, K. et al. 2002, PASJ, 54, 315

Osterbrock, D. E. \& Pogge, R. W. 1985, ApJ, 297, 166

Poggianti, B. M. 1997, A\&AS, 122, 399

Ratnatunga, K. U., Griffiths, R. E., \& Ostrander, E. J. 1999, AJ, 118, 86

Sarajedini, V. L., Green, R. F., Griffiths, R. E., \& Ratnatunga, K. 1999, ApJS, 121, 417

Sarajedini, V. L., Gilland, R. L., Kasm, C. 2003, ApJ, in press (astro-ph/0308383)

Schmidt, M. et al. 1998, A\&A, 329, 495

Tanaka, I., Yamada, T., Aragón-Salamanca, A., Kodama, T., Miyaji, T., Ohta, K., \& Arimoto, N. 2000, ApJ, 528, 123

Tremaine, S. et al. 2002, ApJ, 574, 740

Ueda, Y., Akiyama, M., Ohta, K., \& Miyaji, T. 2003, ApJ, 598, 886

Valtchanov, I., Pierre, M., \& Gastaud, R. 2001, A\&A, 370, 689

Veilleux, S. \& Osterbrock, D. E. 1987, ApJS, 63, 295

Waskett, T. J. et al. 2003, MNRAS, 341, 1217

Wilkes, B. J. 2000 in Allen's Astrophysical Quantities, Fourth Edition, ed. A.N. Cox (New York:Springer), p585

This 2-column preprint was prepared with the AAS LATEX macros v5.2. 